\title{
Location always matters: how to improve performance of dynamic networks?
}

\author{
Michael Segal \\ Department of Communication Systems Engineering \\ Ben-Gurion University of the Negev, Israel \\ Email:segal@bgu.ac.il
}

I N OUR talk we will focus on networks with no predefined infrastructure (ad-hoc networks, sensor networks, vehicular networks). There are many optimization problems derived from the context of such networks including power assignment mechanisms, scheduling, data gathering, etc. We will discuss various techniques tacking these problems emphasizing the importance of mobile nodes locations and its influence on the tightness of the solutions.

In particular, we consider the following scenarios.

- Wireless Sensor Network. A wireless sensor network (WSN) consists of $n$ wireless sensor nodes, $S=$ $\left\{s_{1}, \ldots, s_{n}\right\}$, distributed in some area $A$. These nodes perform monitoring tasks and periodically report to a base station $r$ which is located somewhere within the area $A$ (we consider different locations throughout the paper). During the report phase, the sensor nodes propagate a message to the base station through a data collection tree, $T_{S}=\left(S \cup\{r\}, E_{S}\right)$, rooted at $r$. We consider data collection with aggregation, where every node $s \in S$ forwards a single unit size report message to its parent. The message holds an accumulated information collected from a subtree of $T_{S}$ rooted at $s$. An example of this scenario can be found in temperature monitoring systems for fire prevention, intrusion detection, seismic readings, etc. Minimizing the energy requirement is one of the primary optimization objectives when deploying a WSN due to the very low battery reserves at the sensor nodes and the high costs that are associated with replacing these batteries (if at all possible). The second measure that we are interested in is transport capacity, $D\left(T_{S}\right)$, of the data collection tree $T_{S}$. Another critical aspect in the design of a WSN is the hop-diameter of $T_{S}$. We consider different approaches of $T_{S}$ construction including: shortcutting Minimum Spanning Tree (MST) [1], identification of balance nodes [1], centroid-based constructions [2], $(r, d)$-index constructions [2].

- Wireless Ad-hoc Network. A wireless ad-hoc network consists of transceivers (nodes) that are located in the plane and communicate by radio. In contrast to wired networks, wireless ad-hoc networks have no fixed communication backbone. The temporary physical topology of the network is determined by the relative disposition of the wireless nodes and the transmission range assignment of each of the nodes. The combination of these two factors produces a directed communication graph where the nodes correspond to the transceivers and the edges correspond to the communication links. The topology of the induced communication graph has a strong effect on the routing algorithms' efficiency. In this talk we will discuss one of the key properties of the induced communication graph - energy stretch factor [3], [4]. Let $\gamma_{u, v}$ be the minimum energy required to send a message from $u$ to $v$ (using other nodes if necessary). The energy spanner is aimed at minimizing the energy stretch factor $t_{E}$ of the induced communication graph, that is, for any $u, v$, the energy required to propagate a message from $u$ to $v$ is at most $t_{E} \cdot \gamma_{u, v}$.

- Vehicular Ad-Hoc Network. Vehicular ad-hoc network (VANET) is a promising branch of traditional MANET. VANET is designed to provide wireless communication between vehicles and between vehicles and nearby roadside equipment. This communication intends to improve both safety and comfort on the road. VANET has a number of difficulties regarding the traditional MANET. Due to the dynamic nature of VANET environments, configuration is always changing, where links may appear and disappear very quickly and vehicle density is constantly changing. In this talk, we also will discuss self-organizing hierarchical topology to serve as the infrastructure for beacon dissemination process in VANET by carefully partitioning the network into geographically optimized clusters with chosen clusterheads [5].

\section{REFERENCES}

[1] J. Crowcroft, M. Segal, and L. Levin, "Improved structures for data collection in static and mobile wireless sensor networks," Journal of Heuristics, vol. 21, no. 2, pp. 233-256, 2015.

[2] V. Milyeykovski, M. Segal, and V. Katz, "Using central nodes for efficient data collection in wireless sensor networks," Computer Networks, vol. 91 pp. 425-437, 2015.

[3] H. Shpungin and M. Segal, "Near-optimal multicriteria spanner constructions in wireless ad hoc networks," IEEE/ACM Transansactions on Networking, vol. 18, no. 6, pp. 1963-1976, 2010.

[4] _ _ "Improved multicriteria spanners for ad-hoc networks under energy and distance metrics," ACM Transactions on Sensor Networks, vol. 9 , no. 4, p. 37, 2013.

[5] Y. Allouche and M. Segal, "A cluster-based beaconing approach in vanets: Near optimal topology via proximity information," ACM Mobile Networks and Applications, vol. 18, no. 6, pp. 766-787, 2013. 